\title{
24. Konservativ behandelte Blasenverletzungen bei schweren Schädel-Hirntraumen
}

\author{
R. Engetiking* und K. E. Richard-Köln
}

\section{Conservatively Treated Bladder Injuries in Cases of Severe Skull and Brain Trauma}

Summary. In combined traumas-especially severe skull and brain trauma or injuries to internal organs - the care of a bladder injury may take second place. On the example of two severe skull and brain traumas with pelvic fracture and bladder injury it was shown that exceptionally even an accurate diagnosis of the bladder injury may be omitted and treatment confined to transurethral drainage by indwelling catheter. The provisos are absence of abdominal symptoms, absence of any major urine extravasation, careful and continuous surveillance, antibiotics and secure urine drainage. The possibility of serious consequences of inadequate urine drainage was shown in another patient with skull and brain trauma and initial major haematuria without pelvic fracture who suffered a two-stage rupture of the bladder after accidental removal of the indwelling catheter.

Zusammenfassung. Bei Kombinationstraumen - insbesondere schweren Schädel-Hirntraumen oder inneren Organverletzungen - kann die Versorgung einer Blasenverletzung in den Hintergrund treten. Am Beispiel zweier schwerer SchädelHirntraumen mit Beckenbruch und Blasenverletzung konnte gezeigt werden, daß in Ausnahmefällen sogar auf eine genauere Diagnostik der Blasenverletzung verzichtet und die Behandlung derselben auf eine transurethrale Harnableitung mittels Dauerkatheter beschränkt werden kann. Voraussetzungen sind: Fehlende abdominale Symptomatik, Fehlen eines größeren Harnextravasates, sorgfältige und kontinuierliche Utberwachung, Antibiotica und sichere Harnableitung.

Daß eine ungenügende Harnableitung verhängnisvolle Folgen haben kann, zeigte sich bei einem weiteren Verletzten mit Schädel-Hirntrauma und initialer Makrohämaturie ohne Beckenbruch, bei dem es nach unbeabsichtigter Entfernung des Dauerkatheters zu einer zweizeitigen Blasenruptur kam.

Präsident: Ich glaube, daß wir mit den bisher gehaltenen Vorträgen einen Streifzug durch die gesamte Problematik haben erleben können. Das heißt nicht, daß nicht noch wesentliche Fragen offen geblieben sind. Insofern hoffen wir nun, von der folgenden Forums-Diskussion auch anhand der im Kongreßführer eingehefteten Meldekarten und der zu stellenden Fragen zu einer weiteren Abklärung zu kommen. Ich darf die Herren, die noch nicht zu Wort gekommen sind, bitten, ihr Referat abzugeben und zu Protokoll zu geben. Es wird im Laufe der Diskussion möglich sein, daß Herr Kollege Kremer als Diskussionsleiter den einen oder anderen Vortragenden bittet, in zwei oder drei Sätzen seinen Standpunkt zu formulieren.

Ich darf die Diskussionsteilnehmer, die durchwegs auch Hauptvortragende waren, besonders begrüßen, insbesondere auch Herrn Kollegen Lutz, der sich als Anaesthesist zur Verfügung gestellt hat, um über den Grenzbereich hinausgehend die Fragen von seiner Warte aus mit beleuchten zu können. Damit darf ich die Diskussionsführung an Herrn Kollegen Kremer übergeben. 